\title{
Multifractal analysis of electron images of fossil coal surface
}

\author{
Vasiliy Malinnikov ${ }^{1 *}$, Valeriy Zakharov ${ }^{2}$, Denis Uchaev $^{1}$, Dmitry Uchaev ${ }^{1}$ and Olga \\ Malinnikova $^{2}$ \\ ${ }^{1}$ Moscow State University of Geodesy and Cartography, Department of Space Monitoring and \\ Ecology, Moscow, Russia \\ ${ }^{2}$ Institute of Comprehensive Exploitation of Mineral Resources Russian Academy of Sciences, \\ Moscow, Russia
}

\begin{abstract}
Annotation. The multifractal spectra of electronic images of fossil coal surfaces obtained from outburst-hazardous zones, outburst-nonhazardous (quiet) zones and outburst zones are studied. It is shown that the structural organization of coal surface elements can be represented by a multifractal with a corresponding $f(\alpha)$-spectrum of fractal dimensions, which is suitable to determine quantitative difference in microstructures of coal specimens from outburst-hazardous and outburst-nonhazardous beds. Coals from outburst-hazardous and outburst-nonhazardous beds differ in the direction of skewness of multifractal spectra characterizing their surface structure. If the $f(\alpha)$-spectrum is skewed to the right, then the analyzed coal specimen belongs to an outburst-hazardous bed, and on the contrary, if $f(\alpha)$ is skewed to the left, then it can be concluded that the coal specimen most likely belongs to an outburst-nonhazardous bed.
\end{abstract}

\section{Introduction}

Currently, fossil coal is widely studied using the scanning electron microscopy (SEM) for direct multi-scale visualization of micro- and nanostructures. SEM microscopes have some advantages over light microscopes [1, 2]:

- great depth of focus, which allows analyzing both polished sections and relief surfaces;

- high image magnification: the ultimate magnification of the up-to-the-minute optical microscopes is limited to 2,000 times while SEM microscopes offer magnification to 1,000,000 times;

- sharp contrast imaging of multi-phase specimens since the image contrast in scanning electron microscopes is conditioned by an atomic number of a substance.

Supermolecular structure parameters estimated by means of scanned coal surface images (SCSI) are used to analyze processes of coal formation and the related methane release factor of coal, to calculate coalbed methane release during mining and gas drainage, to predict gas-dynamic events in coal mines etc. Naturally, the researchers search and

* Corresponing author: malinnikov@mail.ru 
develop new methods and techniques for automated interpretation of SCSI with the maximized correction for the probabilistic spectral and spatial structure of bituminous coal. The analysis of fractal and multifractal properties of SCSI is a promising trend in the geophysics inasmuch as it takes into account the fact that the energy spectrum of scanned coal surface images decreases as the spatial frequency increase by power law [3]. Such spectrum is invariant under multiplicative change in scale. The ideal fractal images possess no such notions as a typical size or a characteristic spatial frequency: what happens in a spatial-frequency interval can occur at any scaled frequency range. On the other hand, real image can be assumed as a fractal only within a certain range of spatial frequencies. As a rule, this range is very limited and hardly detectable due to instability of energy spectrum estimates obtained from the images, and owing to the influence of a transfer function of the specific image visualization system. For example, in electron microscopy imaging of microstructures at a tolerable contrast, the frequency response characteristic defines frequency bands visualized at the maximum and minimum contrast. This being the case, the spatial spectral features and typical scales fail to describe real characteristics of an image. It should be taken into account that the transition to a new scale for some discrete image is only possible in case of scale decrease [4].

The purpose of this work is to demonstrate results of multifractal analysis of images of fossil coal surfaces, aimed to identify differences in the spatial structure of outbursthazardous and outburst-nonhazardous coals.

\section{Methodology of research}

The methodology of research included five successive stages.

1. Visual interpretation of original electronic images to pre-identify objects, to eliminate noises, blur and degradation, to highlight boundaries of irregularities, ill-defined regular components etc.

Images of scanning electron microscopy (SEM images) of coal specimens are usually characterized with a high level noise from a number of sources [5]. The paper [6] shows that the Gaussian distribution can be used to approximate noise in SEM images, which appreciably complicates further interpretation due to considerable difference between the values of characteristics obtained using images with and without the Gaussian noise.

In this paper, the multi-scale filter for improving the image quality in the Chebyshev moment domain was used to suppress the noise of SEM images of coal specimens [7]. This filter has already demonstrated its effectiveness in suppressing noise in coal specimen images obtained by SEM [8].

2. Exploratory analysis of investigated SEM images, including:

- assessment of statistical law and characteristics of grey levels in SEM images.

- formation of two-dimensional spatial Fourier spectra and their informative features for analyzed images or sub-images.

During the image analysis, we used power spectrum or power spectrum in log-log axes to calculate the informative features of spatial spectrum (SS) listed below:

- aspect $A$ characterizing elongation of SS: $A=l_{\max } / l_{\min }$, where $l_{\min }$ and $l_{\max }$ are shortest and longest sides of a outlined spectrum, respectively;

- SS connection parameter, which is equal to one for simply connected spectra and to zero for multiply connected spectra;

- slopes of one-dimensional sections of SS in different directions;

- minimum, maximum and average values and dispersion;

- central moments;

- variation coefficient, excess and asymmetry. 
3. Checking the hypothesis about a multifractal nature of coal surface SEM images or sub-images.

The hypothesis about a multifractal nature of grey-level SEM images of coal surface was checked using a number of criteria:

- The energy spectrum of the images decreases as the spatial frequency increases by power law. Such spectrum is invariant under multiplicative variation in scale [9].

- Statistical distribution of grey levels for the images is a near to log-normal [10].

4. Calculation and analysis of multifractal characteristics for investigated SEM images or sub-images.

This stage involves calculation of multifractal spectra $D(q)$ и $f(\alpha)$ and derived characteristics by means of local-global multifractal analysis of digital images [11]. Evaluation and analysis of multifractal characteristics of the investigated images were processed in GEO-PC environment developed at MIIGAIK. Further, informative multifractal characteristics were investigated in order to estimate their usefulness to differ images of coals obtained from outburst-hazardous and outburst-nonhazardous beds, as well as coals taken from outbursts (Table 1).

Table 1. List of the most informative multifractal characteristics.

\begin{tabular}{|l|c|}
\hline \multicolumn{1}{|c|}{ Multifractal characteristic } & Calculation formula \\
\hline$\Delta_{q} D-$ order parameter & $\Delta_{q} D=D_{1}-D_{q}(q>>1)$ \\
\hline $\begin{array}{l}\alpha_{\min }-\text { minimum value of Hölder exponents } \\
\alpha\end{array}$ & $\begin{array}{c}\alpha_{\min }==_{1 \leq i \leq W, 1 \leq j \leq H} \alpha_{i, j}, \\
\text { where } W \text { and } H-\text { are width and height } \\
\text { of the analyzed image or its local region }\end{array}$ \\
\hline $\begin{array}{l}\alpha_{\max }-\text { maximum value of Hölder exponents } \\
\alpha\end{array}$ & $\begin{array}{c}\alpha_{\max }=\max _{1 \leq i \leq W, 1 \leq j \leq H} \alpha_{i, j} \\
\text { where } W \text { and } H-\text { are width and height } \\
\text { of the analyzed image or its local region }\end{array}$ \\
\hline $\begin{array}{l}f\left(\alpha_{\min }\right)-\text { fractal dimension of the image } \\
\text { subset characterized by } \alpha_{\min }\end{array}$ & $f\left(\alpha_{\min }\right)=\inf _{q \in R}\left\{\alpha_{\min } q-\tau(q)\right\}$ \\
\hline $\begin{array}{l}f\left(\alpha_{\max }\right)-\text { fractal dimension of the image } \\
\text { subset characterized by } \alpha_{\max }\end{array}$ & $f\left(\alpha_{\max }\right)=\inf _{q \in R}\left\{\alpha_{\max } q-\tau(q)\right\}$ \\
\hline $\begin{array}{l}\Delta \alpha-\text { range of Hölder exponent values } \alpha \\
(\text { width of } f(\alpha))\end{array}$ & $\Delta \alpha=\alpha_{\max }-\alpha_{\min }$ \\
\hline $\begin{array}{l}S_{L}-\text { area of a figure under the left branch of } \\
f(\alpha)\end{array}$ & $S_{L}=\int_{\alpha_{\min }}^{\alpha_{0}} f(\alpha) d \alpha$ \\
\hline $\begin{array}{l}S_{R}-\text { area of a figure under the right branch } \\
\text { of } f(\alpha)\end{array}$ & $S_{R}=\int_{\alpha_{0}}^{\alpha_{\max }} f(\alpha) d \alpha$ \\
\hline$S_{f}-$ area of a figure under $f(\alpha)$ & $S_{f}=S_{R}+S_{L}$ \\
\hline$r-$ relative asymmetry coefficient for $f(\alpha)$ & $r=\frac{\alpha_{0}-\alpha_{\min }}{\alpha_{\max }-\alpha_{0}}=\frac{\Delta_{\mathrm{L}} \alpha}{\Delta_{\mathrm{R}} \alpha}$ \\
\hline $\begin{array}{l}R-\text { normalized asymmetry coefficient for } \\
f(\alpha)\end{array}$ & $R=\frac{\Delta_{\mathrm{L}} \alpha-\Delta_{\mathrm{R}} \alpha}{\Delta_{\mathrm{L}} \alpha+\Delta_{\mathrm{R}} \alpha}=\frac{\Delta_{\mathrm{L}} \alpha-\Delta_{\mathrm{R}} \alpha}{\Delta \alpha}$ \\
\hline
\end{tabular}

5. Analysis of results of multifractal analysis of coal surface SEM images. 
The final stage of the proposed procedure consists in an expert analysis of the obtained multifractal parameters of coal surface SEM images.

\section{Source Data}

In this study, imaging of coal specimen surfaces was performed using scanning electron microscopes JEOL JSM 5910-LV and Jeol-6610-LV. The spatial resolution of the microscopes is more than 10 and $100 \mathrm{~nm}$ for secondary and reflected electrons, respectively. Low-energy secondary electrons are used in imaging surface topography. Natural-shape coal specimens were placed in a work camera via a gate. In the mode of registration of secondary electrons, the work camera was vacuumized (with $>10-6 \mathrm{~mm}$ hg vacuum). Secondary electrons were recorded by a standard detector, which a type of a sweeping-field photomultiplier tube connected to scintillator.

As the source data for this study was IPKON collection of coal specimens from the Zapolyarnaya mine (Vorkuta) and the Kirov mine (Leninsk-Kuznetsk), obtained from outburst-nonhazardous zones, outburst-nonhazardous zones and outburst zones. For our research, we analyzed microstructure of coal surface in the images magnified 1,000 times which showed coal grains with a characteristic size from .5 to a few microns. Methane can desorb from such grains, diffuse and flow in fractures as free gas [12].

\section{Research Results}

Results of Fourier analysis demonstrated that the different irregularities in the coal surface microstructure are described by spatial spectra with different shape and statistical characteristics. At the same time, the spectrum curve for all analyzed specimens can be approximated by a power function, which proves the hypothesis about the fractal nature of the analyzed images.

The statistical analysis of the analyzed images shows that their grey-level distributions have peaks and a distinct positive asymmetry. Approximations of grey-level distributions showed that they could be described by a log-normal law with $95 \%$ confidence level. This important outcome is a consequence of a multifractal structure of analyzed coal surface images.

Thus, both tested criteria showed the feasibility of multifractal analysis and the possibility of correct interpretation of results of the multifractal analysis. On this basis, for each image, multifractal spectra $D(q)$ and $f(\alpha)$ with characteristics derived from these spectra were calculated. Then, for each multifractal characteristic, it was analyzed the possibility of each multifractal characteristic to serve as a disciminative feature to differentiate outburst-nonhazardous and outburst-nonhazardous coal beds using images of coal specimens from these beds. As a result of the analysis, it was revealed that the normalized asymmetry coefficient $R$ for $f(\alpha)$-spectrum has the greatest deciphering potential. After calculation of $R$ values for all analyzed coal specimens, we found that the surface structure of outburst-hazardous coal specimens can be characterized by a fractal dimension spectrum with positive skewness whereas the surface structure of outburstnonhazardous coal specimens is characterized by a symmetrical fractal spectrum (with negative skewness close to zero) (figure 1). This result is verified for nearly $80 \%$ of all test coal specimens.

For each of our groups of coal specimens, ranges of the most likely values for $R$ were also obtained (shown as dashed lines in Fig. 1). As can be seen from Fig. 1, approximately $60 \%$ of all estimates for $R$ obtained for coals from outburst-hazardous zones, outburstnonhazardous zones and outburst zones fall into non-overlapping ranges $[-0.845,-0.505]$, 
$[-0.005,0.215]$ and $[-0.300,0]$, respectively. Thus, we can assume that the asymmetry of fractal dimension spectra characterizing coal surface structure can be used as a criterion for verification of coal susceptibility to gas-dynamic events.

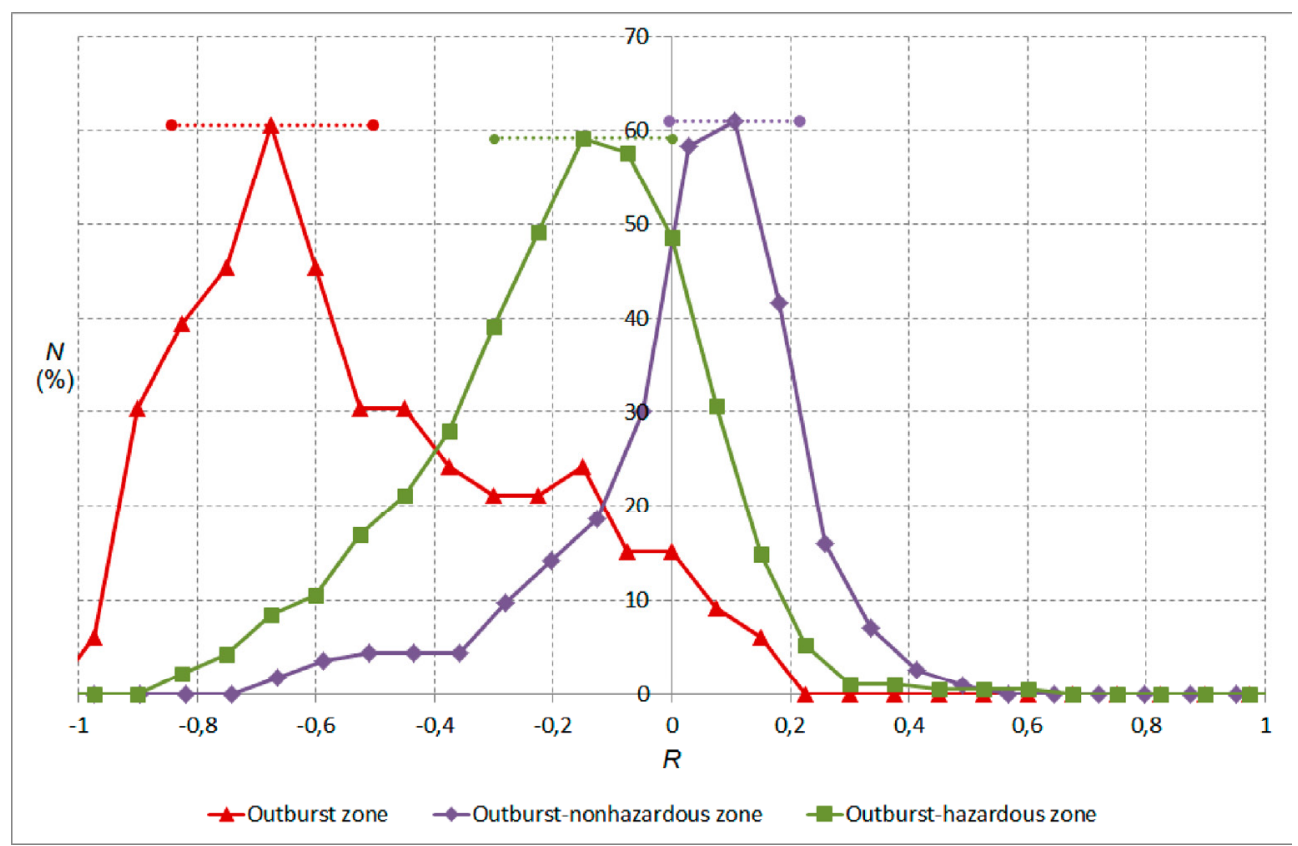

Fig. 1. Number $N(\%)$ of estimates for $R$, calculated for analyzed coal specimens. The distance between centers of bins and the bin width for each histogram were chosen so that about $60 \%$ of all estimates for $R$ fall into the ranges of the most likely values for $R$.

\section{Conclusions}

Based on the implemented research, it has been concluded that the multifractal approach is efficiently applicable to investigations of fossil coal damage and can be used to estimate the connection between the fractal properties of damaged coal structure and coal susceptibility to gas-dynamic events. As our investigations have shown, there is a certain relationship between the degree of coal susceptibility to outbursts and the asymmetry of multifractal spectra calculated using coal specimen images. The obtained criterion relations can be used in the preliminary prediction of coal susceptibility to gas-dynamic events by the structural factor; however, these relations are advisable to refine through analyzing a wider class of different scale electron images of coal surface.

The development of the technique for exploratory multifractal analysis, as well as the technique and algorithm for calculating multifractal characteristics, was conducted in the framework of the state task of the Ministry of Education and Science of the Russian Federation (No. 5.6470.2017/8.9). The remaining studies were supported by the Russian Science Foundation, Project No. 16-05-00816.

\section{References}

1. W. Zhou, Z.L. Wang, Scanning microscopy for nanotechnology: techniques and applications (BINOM: Laboratory of Knowledge, 2014).

2. L. Chen, J. Xu, J. Chen, Science China Earth Sciences. 58(10), 1768-1778 (2015). 
3. V.A. Malinnikov, O.N. Malinnikova, V.N. Zakharov, Procs of XXVII International School of Academician Khristianovch «Deformation and destruction of materials with defects and dynamic phenomena in rocks and excavations», 158-162 (2017).

4. B.N. Grudin, V.S. Plotnikov, N.A. Smolyaninov, Optoelectronics, Instrumentation and Data Processing, 46(3), 215-221 (2010).

5. D.V. Nesterenko, Computer Optics, 35(2), 166-174 (2011).

6. N. Marturi, S. Dembélé, N. Piat, Scanning. 36(4), 419-429 (2014).

7. Dm.V. Uchaev, D.V. Uchaev, V.A. Malinnikov, Proc. SPIE, 9875, 987512 (2015).

8. O.N. Malinnikova, V.A. Malinnikov, Dm.V. Uchaev, D.V. Uchaev, Procs of XXVII International School of Academician Khristianovch «Deformation and destruction of materials with defects and dynamic phenomena in rocks and excavations», 134-139 (2016).

9. M. Schroeder, Fractals, Chaos, Power Laws: Minutes from an Infinite Paradise («RHD», 2001).

10. S.V. Bozhokin, D.A. Parshin, Fractals and multifractals («RHD», 2001).

11. V.A. Malinnikov, D.V. Uchaev, Dm.V. Uchaev, Izvestia vuzov «Geodesy and aerophotography», 4, 64-68 (2010).

12. O.N. Malinnikova, V.N. Odintsev, V.A. Trofimov, Methane/GIAB, SI 11, 189-204 (2009). 